University of Zurich

Department of Economics

Working Paper Series

ISSN 1664-7041 (print)

ISSN1664-705X(online)

Working Paper No. 21

\title{
On the Equivalence of Bayesian and Dominant Strategy Implementation in a General Class of Social Choice Problems
}

Jacob K. Goeree and Alexey Kushnir

July 2011 


\title{
On THE EquiVALENCE OF BAYESIAN AND Dominant Strategy Implementation in a General Class of Social Choice Problems
}

\author{
Jacob K. Goeree and Alexey Kushnir*
}

July 25, 2011

\begin{abstract}
We consider a standard social choice environment with linear utilities and independent, one-dimensional, private values. We provide a short and constructive proof that for any Bayesian incentive compatible mechanism there exists an equivalent dominant strategy incentive compatible mechanism that delivers the same interim expected utilities for all agents. We demonstrate the usefulness and applicability of our approach with several examples. Finally, we show that the equivalence between Bayesian and dominant strategy implementation breaks down when utilities are non-linear or when values are interdependent, multi-dimensional, or correlated.
\end{abstract}

${ }^{*}$ Chair for Organizational Design, Department of Economics, University of Zürich, Blümlisalpstrasse 10, CH8006, Zürich, Switzerland. We gratefully acknowledge financial support from the European Research Council (ERC Advanced Investigator Grant, ESEI-249433). We would like to thank Benny Moldovanu for pointing us towards the Gutmann et al. (1991) paper and Angel Hernando-Veciana and Konrad Mierendorff for useful discussions. 


\section{Introduction}

This paper revisits Bayesian and dominant strategy implementation in standard social choice environments with linear utilities and independent, one-dimensional, private values. We prove that for any Bayesian incentive compatible (BIC) mechanism there exists an equivalent dominant strategy incentive compatible (DIC) mechanism that delivers the same interim expected utilities for all agents. This equivalence result is surprising and valuable in that dominant strategy implementation has important advantages over Bayesian implementation. In particular, dominant strategy implementation is robust to changes in agents' beliefs and does not rely on the assumptions of a common prior and equilibrium play.

In an inspiring recent contribution, Manelli and Vincent (2010) proved equivalence of Bayesian and dominant strategy implementation for single-unit, private-value auctions. In this setting, each agent's interim expected utility is determined (up to a constant) by the conditional expected probability of winning the object. Manelli and Vincent's equivalence result thus implies that for any BIC mechanism there is a DIC mechanism that yields the same conditional expected probabilities with which each alternative is selected. However, Gershkov, Moldovanu, and Shi (2011) provide a counter example to show that this stronger result does not extend to more general social choice environments with at least three alternatives. Their negative conclusion does not contradict the results of this paper because of the different notion of equivalence we employ. In particular, we define two mechanisms to be equivalent if agents' interim expected utilities are the same, which is generally less demanding than the requirement that the conditional expected probabilities with which each alternative occurs are the same. We illustrate the difference within the context of the counter example proposed by Gershkov, Moldovanu, and Shi (2011) and demonstrate that BIC-DIC equivalence continues to hold.

Our method of proof is based on a theorem due to Gutmann et al. (1991), which was introduced to the economics literature by Gershkov, Moldovanu, and Shi (2011). The theorem states that for any bounded, non-negative function of several variables that generates monotone, one-dimensional marginals there exist a non-negative function that is monotone in each coordinate and which produces the same one-dimensional marginals and respects the same bounds. The proof is constructive and shows how the desired function can be found as a solution to a minimization problem. Gershkov, Moldovanu, and Shi (2011) use this theorem to establish BIC-DIC equivalence for social choice environments with two alternatives and for symmetric, single-unit auctions with an arbitrary number of bidders. The restriction to two alternatives or symmetry is necessary because the Gutmann et al. (1991) theorem concerns a single function with given marginals. In a two-alternative social choice setting this single function can describe 
the probability with which one of the alternatives occurs (while the other alternative occurs with complementary probability). Likewise, in a symmetric auction setting, this single function can describe the probability with which, say, bidder 1 wins, while the remaining probability is evenly distributed among the other bidders. The original Gutmann et al. (1991) theorem, however, cannot be applied to general social choice environments or asymmetric auctions.

This paper generalizes the results of Manelli and Vincent (2010) and Gershkov, Moldovanu, and Shi (2011) by considering social choice environments with an arbitrary number of players and alternatives and arbitrary type distributions. We prove an extension of the Gutmann et al. (1991) theorem, which involves minimizing a quadratic functional of several functions satisfying certain boundary and marginal constraints. We provide a simple procedure to construct for any BIC mechanism an equivalent DIC mechanism and illustrate our construction with several examples. Finally, we show that the assumptions under which we derive BIC-DIC equivalence, i.e. linear utilities and independent, one-dimensional, private values, are not only sufficient but also necessary. Using simple examples, we demonstrate that BIC-DIC equivalence fails when one of these conditions is not met.

Within the context of auction design the implications of our equivalence result can be highlighted as follows. BIC-DIC equivalence implies that any optimal auction (in terms of efficiency or revenue) can be implemented using a dominant strategy mechanism such as the second-price auction. In other words, nothing can be gained from designing more intricate auction formats with possibly more complex Bayes-Nash equilibria. This holds not only for single-unit auctions but also for multi-unit auctions with homogeneous or heterogeneous goods, combinatorial auctions, and the like, as long as bidders' private values are one-dimensional and independent and utilities are linear.

Once we relax the assumptions underlying our main theorem, however, Bayesian implementation may have advantages over dominant strategy implementation. For example, we show that efficiency may be strictly higher under BIC implementation when values are interdependent. Likewise, with multi-dimensional values, BIC mechanisms may result in higher revenues than can be attained by any DIC mechanism.

\subsection{Related Literature}

There exists an extensive literature on implementation of social choice rules. Gibbard (1973) and Satterthwaite (1975) show that only dictatorial choice rules are dominant strategy im-

plementable if preferences are unrestricted and there are at least three alternatives. Roberts (1979) provides a complete characterization of social choice rules implementable in dominant 
strategies for an environment with quasi-linear preferences and at least three alternatives.11 Jehiel et al. (2008) extend this result to settings with multi-dimensional types and additively separable utilities. For generic environments with multi-dimensional signals and interdependent valuations, however, Jehiel et al. (2006) show that there are no non-trivial deterministic social choice functions that are ex post implementable. Unlike these papers, we do not study whether a certain social choice rule is Bayesian or dominant strategy implementable. Rather we provide a link between Bayesian and dominant strategy implementation.

Along this line, Mookherjee and Reichelstein (1992) provide sufficient conditions for BIC social choice rules to be dominant strategy implementable and they identify environments where these conditions are necessary. In contrast, we are not concerned with the implementation of a given social choice rule but rather construct for any social choice rule that is Bayesian implementable another social choice rule that is dominant strategy implementable and that delivers the same interim expected payoffs for all agents.

A main focus of the mechanism design literature concerns the implementation of efficient mechanisms. Green and Laffont (1977) prove that any efficient direct mechanism in which truthful revelation is a dominant strategy is necessarily a Groves mechanism. d'Aspremont and Gérard-Varet (1979), Laffont and Maskin (1979), and subsequently Williams (1999) extend this result to Bayesian environments by showing that any efficient BIC mechanism is payoff equivalent to some Groves mechanism. Jehiel and Moldovanu (2001) demonstrate that Bayesian implementation of efficient choice functions is impossible in generic settings with multidimensional signals and interdependent valuations. This paper differs in that our equivalence result applies to every BIC mechanism, not just efficient ones.

Several papers study the implementation of efficient BIC mechanisms satisfying certain additional constraints, such as budget balance and individual rationality. For instance, d'Aspremont and Gérard-Varet (1979) explicitly construct mechanisms that are efficient and ex post budget balanced (although they may violate individual rationality). Myerson and Satterthwaite (1983) consider a classical bargaining model with incomplete information and show that generally there is no BIC mechanism that is interim individual rational and ex ante budget balanced. Makowski and Mezzetti (1994) prove that for a given efficient interim individual rational and ex ante budget balanced DIC mechanism, there exists a BIC mechanism that is efficient, interim individually rational, and ex post budget balanced. As a contribution to this literature our equivalence result suggests that Bayesian and dominant strategy implementation are equivalent if only ex ante or interim constraints are imposed.

\footnotetext{
${ }^{1}$ Laffont and Maskin (1982) provide a complete characterization of dominant strategy implementable social choice rules for the case of two alternatives. Lavi, Mualem, Nisan (2009) provide two alternative proofs of Roberts' result.
} 
Our analysis makes use of a payoff equivalence principle for BIC and DIC mechanisms. The earliest development of this principle for DIC mechanisms can be found in Green and Laffont (1979) and Laffont and Maskin (1979, 1980), and for BIC mechanisms in Myerson (1981). The most general conditions under which the principle holds can be found in recent contributions by Krishna and Maenner (2001), Milgrom and Segal (2002), Jehiel and Moldovanu (2001), and Heydenreich et al. (2009). Jehiel et al. (1996, 1999) also establish payoff equivalence for auctions with multi-dimensional types and externalities.

The paper is organized as follows. Section 2 presents the social choice environment. We prove our main BIC-DIC equivalence result in Section 3. In Section 4 we illustrate our approach with some examples and compare it to related work. In Section 5 we demonstrate that linear utilities and independent, one-dimensional, private values are not only sufficient but also necessary conditions for BIC-DIC equivalence. Section 6 concludes. The Appendix contains some proofs.

\section{Model}

We consider an environment with a finite set $\mathcal{I}=\{1,2, \ldots, I\}$ of risk-neutral agents and a finite set $\mathcal{K}=\{1,2, \ldots, K\}$ of social alternatives. Agent $i$ 's utility in alternative $k$ equals $u_{i}^{k}\left(x_{i}, t_{i}\right)=a_{i}^{k} x_{i}+c_{i}^{k}+t_{i}$ where $x_{i}$ is agent $i$ 's private type, $a_{i}^{k}, c_{i}^{k} \in \mathbb{R}$ are constants with $a_{i}^{k} \geq 0$, and $t_{i} \in \mathbb{R}$ is a monetary transfer. Agent $i$ 's type $x_{i}$ is distributed according to probability distribution $\lambda_{i}$ with support $X_{i}$, where the type space $X_{i} \subseteq \mathbb{R}$ can be any (possibly discrete) subset of $\mathbb{R}$. Note that types are one-dimensional and independent. Let $X=\prod_{i \in \mathcal{I}} X_{i}$ and $\lambda=\prod_{i \in \mathcal{I}} \lambda_{i}$.

Without loss of generality we consider only direct mechanisms (e.g. Mas-Colell, Whinston, and Green, 1995, Ch. 23; Fudenberg and Tirole, 1991, Ch. 7), characterized by $K+I$ functions, $\left\{q^{k}(\mathbf{x})\right\}_{k \in \mathcal{K}}$ and $\left\{t_{i}(\mathbf{x})\right\}_{i \in \mathcal{I}}$, where $\mathbf{x}=\left(x_{1}, \ldots, x_{I}\right) \in X$ is the profile of reports, $q^{k}(\mathbf{x}) \geq 0$ is the probability that alternative $k$ is implemented with $\sum_{k \in \mathcal{K}} q^{k}(\mathbf{x})=1$, and $t_{i}(\mathbf{x})$ is the amount agent $i$ receives. When agent $i$ reports $x_{i}^{\prime}$ and all other agents report truthfully, the conditional expected probability (from agent $i$ 's point of view) that alternative $k$ is chosen is $Q_{i}^{k}\left(x_{i}^{\prime}\right)=$ $E_{\mathbf{x}_{-i}}\left(q^{k}\left(x_{i}^{\prime}, \mathbf{x}_{-i}\right)\right)$ and the conditional expected transfer to agent $i$ is $T_{i}\left(x_{i}^{\prime}\right)=E_{\mathbf{x}_{-i}}\left(t_{i}\left(x_{i}^{\prime}, \mathbf{x}_{-i}\right)\right)$. For later use we define, for $i \in \mathcal{I}$ and $\mathbf{x} \in X$,

$$
v_{i}(\mathbf{x}) \equiv \sum_{k \in \mathcal{K}} a_{i}^{k} q^{k}(\mathbf{x})
$$


with marginals $V_{i}\left(x_{i}\right)=\sum_{k \in \mathcal{K}} a_{i}^{k} Q_{i}^{k}\left(x_{i}\right)$ and the modified transfers

$$
\tau_{i}(\mathbf{x})=t_{i}(\mathbf{x})+\sum_{k \in \mathcal{K}} c_{i}^{k} q^{k}(\mathbf{x})
$$

with marginals $\mathcal{T}_{i}\left(x_{i}\right)=E_{\mathbf{x}_{-i}}\left(\tau_{i}\left(x_{i}, \mathbf{x}_{-i}\right)\right)=T_{i}\left(x_{i}\right)+\sum_{k} c_{i}^{k} Q_{i}^{k}\left(x_{i}\right)$. When agent $i$ 's type is $x_{i}$ and she reports being of type $x_{i}^{\prime}$ her interim expected utility can then be written as

$$
u_{i}\left(x_{i}^{\prime}\right)=V_{i}\left(x_{i}^{\prime}\right) x_{i}+\mathcal{T}_{i}\left(x_{i}^{\prime}\right)
$$

In this paper we restrict attention to Bayesian incentive compatible (BIC) and dominant strategy incentive compatible (DIC) mechanisms. A mechanism is BIC if truthful reporting by all agents constitutes a Bayes-Nash equilibrium. A mechanism is DIC if truthful reporting is a dominant strategy equilibrium. To relate BIC and DIC mechanisms we employ the following notion of equivalence.

Definition 1. Two mechanisms $M$ and $\tilde{M}$ are equivalent if they deliver the same interim expected utilities for all agents.

For single-unit auctions, equivalence implies that $M$ and $\tilde{M}$ yield identical conditional winning probabilities, see Manelli and Vincent (2010). In general, however, our definition of equivalence does not require that the conditional expected probability with which each alternative occurs is the same under both mechanisms.

\section{BIC-DIC Equivalence}

We first consider connected type spaces, i.e. $X_{i}=\left[\underline{x}_{i}, \bar{x}_{i}\right] \subseteq \mathbb{R}$. In this case a mechanism is BIC if and only if (i) for all $i \in \mathcal{I}$ and $x_{i} \in X_{i}, V_{i}\left(x_{i}\right)$ is non-decreasing in $x_{i}$ and (ii) agents' interim expected utilities satisfy

$$
u_{i}\left(x_{i}\right)=u_{i}\left(\underline{x}_{i}\right)+\int_{\underline{x}_{i}}^{x_{i}} V_{i}(t) d t
$$

see, for instance, Krishna and Maenner (2001), Milgrom and Segal (2002), and Jehiel and Moldovanu (2001). Similarly a mechanism is DIC if and only if (i) for all $i \in \mathcal{I}$ and $\mathbf{x} \in X$, $v_{i}\left(x_{i}, \mathbf{x}_{-i}\right)$ is non-decreasing in $x_{i}$ and (ii) agents' utilities can be expressed as

$$
u_{i}\left(x_{i}, \mathbf{x}_{-i}\right)=u_{i}\left(\underline{x}_{i}, \mathbf{x}_{-i}\right)+\int_{\underline{x}_{i}}^{x_{i}} v_{i}\left(t, \mathbf{x}_{-i}\right) d t
$$


see, for instance, Laffont and Masking (1980) and Heydenreich, Müller, Uetz, and Vohra (2009). Hence, with connected type spaces, agents' utilities are determined (up to a constant) by the allocation rule. This allows us to define equivalence in terms of the allocation rule only. Consider two mechanisms $M$ and $\tilde{M}$ and choose transfers such that $u_{i}\left(\underline{x}_{i}\right)=\tilde{u}_{i}\left(\underline{x}_{i}\right)$ for all $i \in \mathcal{I}$, then $M$ and $\tilde{M}$ are equivalent if and only if $V_{i}\left(x_{i}\right)=\tilde{V}_{i}\left(x_{i}\right)$ for all $i \in \mathcal{I}, x_{i} \in X_{i}$.

We now state and prove our main result. Define $\mathbf{v}(\mathbf{x})=A \cdot \mathbf{q}(\mathbf{x})$ with elements $v_{i}(\mathbf{x})$ for $i \in \mathcal{I}$, and let $\|\cdot\|$ denote the usual Euclidean norm: $\|\mathbf{v}(\mathbf{x})\|^{2}=\sum_{i \in \mathcal{I}} v_{i}(\mathbf{x})^{2}$. Throughout we identify functions that are equal almost everywhere with respect to $\lambda$.

Theorem 1. Let $X_{i}$ be connected for all $i \in \mathcal{I}$ and let $(\tilde{q}, \tilde{t})$ denote a BIC mechanism. An equivalent DIC mechanism is given by $(q, t)$, where the allocation rule $q$ solves

$$
\begin{aligned}
& \min _{\left\{q^{k}\right\}_{k \in \mathcal{K}}} E_{\mathbf{x}}\left(\|\mathbf{v}(\mathbf{x})\|^{2}\right) \\
& q^{k}(\mathbf{x}) \geq 0 \quad \forall k, \mathbf{x} \\
& \sum_{k} q^{k}(\mathbf{x})=1 \quad \forall \mathbf{x} \\
& V_{i}\left(x_{i}\right)=\tilde{V}_{i}\left(x_{i}\right) \quad \forall i, x_{i} \\
& E_{\mathbf{X}}\left(q^{k}(\mathbf{x})\right)=E_{\mathbf{X}}\left(\tilde{q}^{k}(\mathbf{x})\right) \quad \forall k
\end{aligned}
$$

and the transfers $t$ follow from

$$
\tau_{i}\left(x_{i}, \mathbf{x}_{-i}\right)=\tilde{\mathcal{T}}_{i}\left(\underline{x}_{i}\right)+v_{i}\left(\underline{x}_{i}, \mathbf{x}_{-i}\right) \underline{x}_{i}-v_{i}\left(x_{i}, \mathbf{x}_{-i}\right) x_{i}+\int_{\underline{x}_{i}}^{x_{i}} v_{i}\left(t, \mathbf{x}_{-i}\right) d t .
$$

Expected transfers are the same under the BIC and DIC mechanisms, i.e. $E_{\mathbf{x}}\left(t_{i}(\mathbf{x})\right)=E_{\mathbf{x}}\left(\tilde{t}_{i}(\mathbf{x})\right)$ for all $i \in \mathcal{I}$, and, hence, so is expected social surplus.

Proof. The main difficulty of the proof is to establish that a solution $v_{i}\left(x_{i}, \mathbf{x}_{-i}\right)$ to (1) is non-decreasing in $x_{i}$, which we do in three steps. First, we consider discrete and uniformly distributed types, then we extend to the continuous uniform types using a discrete approximation, and, finally, we generalize to arbitrary type distributions. The first step, which provides an extension of the Gutmann et. al (1991) theorem, is covered in the main text while the proofs for the more technical second and third steps can be found in the Appendix.

Lemma 1. Suppose, for all $i \in \mathcal{I}, X_{i}$ is a discrete set and $\lambda_{i}$ is uniform distribution on $X_{i}$. Let $\left\{q^{k}\right\}_{k \in \mathcal{K}}$ be a solution to (1) then $v_{i}(\mathbf{x})$ is non-decreasing in $x_{i}$ for all $i \in \mathcal{I}, \mathbf{x} \in X$.

Proof. Let $C(\tilde{q})$ denote the collection of $\left\{q^{k}\right\}_{k \in \mathcal{K}}$ that satisfy the constraints in (1). Note that $C(\tilde{q})$ is non-empty since $\tilde{q} \in C(\tilde{q})$. Furthermore, for discrete $X, C(\tilde{q})$ is a compact and convex subset of $[0,1]^{K|X|}$ and $E_{\mathbf{x}}\left(\|\mathbf{v}(\mathbf{x})\|^{2}\right)$ defines a convex function so existence of a solution to (1) follows from standard arguments. Let $\left\{q^{k}\right\}_{k \in \mathcal{K}}$ be a solution to (1). We need to show that $v_{i}(\mathbf{x})$ is non-decreasing in $x_{i}$ for all $i \in \mathcal{I}$ and $\mathbf{x} \in X$. Suppose, in contradiction, that $v_{j}\left(x_{j}, \mathbf{x}_{-j}\right)>v_{j}\left(x_{j}^{\prime}, \mathbf{x}_{-j}\right)$ for some $j, x_{j}^{\prime}>x_{j}$, and some $\mathbf{x}_{-j}$. Since $\left\{\tilde{q}^{k}\right\}_{k \in \mathcal{K}}$ 
is a BIC mechanism $E_{\mathbf{x}_{-j}}\left(v_{j}\left(x_{j}, \mathbf{x}_{-j}\right)\right)=E_{\mathbf{x}_{-j}}\left(\tilde{v}_{j}\left(x_{j}, \mathbf{x}_{-j}\right)\right)$ is non-decreasing in $x_{j}$. Hence, there exists $\mathbf{x}_{-j}^{\prime}$ for which $v_{j}\left(x_{j}, \mathbf{x}_{-j}^{\prime}\right)<v_{j}\left(x_{j}^{\prime}, \mathbf{x}_{-j}^{\prime}\right)$. Let $\alpha \equiv \varepsilon /\left(v_{j}\left(x_{j}, \mathbf{x}_{-j}\right)-v_{j}\left(x_{j}^{\prime}, \mathbf{x}_{-j}\right)\right)$ and $\alpha^{\prime} \equiv \varepsilon /\left(v_{j}\left(x_{j}^{\prime}, \mathbf{x}_{-j}^{\prime}\right)-v_{j}\left(x_{j}, \mathbf{x}_{-j}^{\prime}\right)\right)$ then, for small $\varepsilon>0$, we have $0<\alpha<1$ and $0<\alpha^{\prime}<1$. Define the perturbations

$$
\begin{gathered}
\mathbf{q}^{\prime}\left(x_{j}, \mathbf{x}_{-j}\right)=(1-\alpha) \mathbf{q}\left(x_{j}, \mathbf{x}_{-j}\right)+\alpha \mathbf{q}\left(x_{j}^{\prime}, \mathbf{x}_{-j}\right), \quad \mathbf{q}^{\prime}\left(x_{j}^{\prime}, \mathbf{x}_{-j}\right)=(1-\alpha) \mathbf{q}\left(x_{j}^{\prime}, \mathbf{x}_{-j}\right)+\alpha \mathbf{q}\left(x_{j}, \mathbf{x}_{-j}\right) \\
\mathbf{q}^{\prime}\left(x_{j}, \mathbf{x}_{-j}^{\prime}\right)=\left(1-\alpha^{\prime}\right) \mathbf{q}\left(x_{j}, \mathbf{x}_{-j}^{\prime}\right)+\alpha^{\prime} \mathbf{q}\left(x_{j}^{\prime}, \mathbf{x}_{-j}^{\prime}\right), \quad \mathbf{q}^{\prime}\left(x_{j}^{\prime}, \mathbf{x}_{-j}^{\prime}\right)=\left(1-\alpha^{\prime}\right) \mathbf{q}\left(x_{j}^{\prime}, \mathbf{x}_{-j}^{\prime}\right)+\alpha^{\prime} \mathbf{q}\left(x_{j}, \mathbf{x}_{-j}^{\prime}\right)
\end{gathered}
$$

and $\mathbf{q}^{\prime}(\mathbf{x})=\mathbf{q}(\mathbf{x})$ for other $\mathbf{x} \in X$. By construction $q^{\prime k}(\mathbf{x}) \geq 0$ and $\sum_{k \in \mathcal{K}} q^{\prime k}(\mathbf{x})=1$ for all $\mathbf{x} \in X$. Also $E_{\mathbf{x}}\left(\mathbf{q}^{\prime}(\mathbf{x})\right)=E_{\mathbf{x}}(\mathbf{q}(\mathbf{x}))$ since $\mathbf{q}^{\prime}\left(x_{j}, \mathbf{x}_{-j}\right)+\mathbf{q}^{\prime}\left(x_{j}^{\prime}, \mathbf{x}_{-j}\right)+\mathbf{q}^{\prime}\left(x_{j}, \mathbf{x}_{-j}^{\prime}\right)+\mathbf{q}^{\prime}\left(x_{j}^{\prime}, \mathbf{x}_{-j}^{\prime}\right)=$ $\mathbf{q}\left(x_{j}, \mathbf{x}_{-j}\right)+\mathbf{q}\left(x_{j}^{\prime}, \mathbf{x}_{-j}\right)+\mathbf{q}\left(x_{j}, \mathbf{x}_{-j}^{\prime}\right)+\mathbf{q}\left(x_{j}^{\prime}, \mathbf{x}_{-j}^{\prime}\right)$. We next show that the perturbations $\mathbf{q}^{\prime}$ also produce the same marginals as $\mathbf{q}$. Rewrite the above perturbations in terms of $\mathbf{v}^{\prime}(\mathbf{x})=A \cdot \mathbf{q}^{\prime}(\mathbf{x})$ :

$$
\begin{gathered}
\mathbf{v}^{\prime}\left(x_{j}, \mathbf{x}_{-j}\right)=(1-\alpha) \mathbf{v}\left(x_{j}, \mathbf{x}_{-j}\right)+\alpha \mathbf{v}\left(x_{j}^{\prime}, \mathbf{x}_{-j}\right), \quad \mathbf{v}^{\prime}\left(x_{j}^{\prime}, \mathbf{x}_{-j}\right)=(1-\alpha) \mathbf{v}\left(x_{j}^{\prime}, \mathbf{x}_{-j}\right)+\alpha \mathbf{v}\left(x_{j}, \mathbf{x}_{-j}\right) \\
\mathbf{v}^{\prime}\left(x_{j}, \mathbf{x}_{-j}^{\prime}\right)=\left(1-\alpha^{\prime}\right) \mathbf{v}\left(x_{j}, \mathbf{x}_{-j}^{\prime}\right)+\alpha^{\prime} \mathbf{v}\left(x_{j}^{\prime}, \mathbf{x}_{-j}^{\prime}\right), \quad \mathbf{v}^{\prime}\left(x_{j}^{\prime}, \mathbf{x}_{-j}^{\prime}\right)=\left(1-\alpha^{\prime}\right) \mathbf{v}\left(x_{j}^{\prime}, \mathbf{x}_{-j}^{\prime}\right)+\alpha^{\prime} \mathbf{v}\left(x_{j}, \mathbf{x}_{-j}^{\prime}\right)
\end{gathered}
$$

and the equal-margin condition as $E_{\mathbf{x}_{-i}}\left(v_{i}^{\prime}\left(x_{i}, \mathbf{x}_{-i}\right)\right)=E_{\mathbf{x}_{-i}}\left(v_{i}\left(x_{i}, \mathbf{x}_{-i}\right)\right)$. For $i=j$ this condition follows from $\alpha\left(v_{j}\left(x_{j}, \mathbf{x}_{-j}\right)-v_{j}\left(x_{j}^{\prime}, \mathbf{x}_{-j}\right)\right)=\alpha^{\prime}\left(v_{j}\left(x_{j}^{\prime}, \mathbf{x}_{-j}^{\prime}\right)-v_{j}\left(x_{j}, \mathbf{x}_{-j}^{\prime}\right)\right)$ when $x_{i}=x_{j}$ or $x_{i}=x_{j}^{\prime}$ while for other values of $x_{i}$ it follows trivially. For $i \neq j$ the condition follows since $\mathbf{v}^{\prime}\left(x_{j}, \mathbf{x}_{-j}\right)+$ $\mathbf{v}^{\prime}\left(x_{j}^{\prime}, \mathbf{x}_{-j}\right)=\mathbf{v}\left(x_{j}, \mathbf{x}_{-j}\right)+\mathbf{v}\left(x_{j}^{\prime}, \mathbf{x}_{-j}\right)$ and $\mathbf{v}^{\prime}\left(x_{j}, \mathbf{x}_{-j}^{\prime}\right)+\mathbf{v}^{\prime}\left(x_{j}^{\prime}, \mathbf{x}_{-j}^{\prime}\right)=\mathbf{v}\left(x_{j}, \mathbf{x}_{-j}^{\prime}\right)+\mathbf{v}\left(x_{j}^{\prime}, \mathbf{x}_{-j}^{\prime}\right)$. Finally,

$$
\begin{aligned}
E_{\mathbf{x}}\left(\left\|\mathbf{v}^{\prime}(\mathbf{x})\right\|^{2}-\|\mathbf{v}(\mathbf{x})\|^{2}\right)= & -\frac{2 \alpha(1-\alpha)}{|X|}\left\|\mathbf{v}\left(x_{j}, \mathbf{x}_{-j}\right)-\mathbf{v}\left(x_{j}^{\prime}, \mathbf{x}_{-j}\right)\right\|^{2} \\
& -\frac{2 \alpha^{\prime}\left(1-\alpha^{\prime}\right)}{|X|}\left\|\mathbf{v}\left(x_{j}^{\prime}, \mathbf{x}_{-j}^{\prime}\right)-\mathbf{v}\left(x_{j}, \mathbf{x}_{-j}^{\prime}\right)\right\|^{2}
\end{aligned}
$$

a contradiction since the right side is strictly negative and $\left\{q^{k}\right\}_{k \in \mathcal{K}}$ solves (1).

Q.E.D.

Lemma 2. Suppose, for all $i \in \mathcal{I}, X_{i}=[0,1]$ and $\lambda_{i}$ is the uniform distribution on $X_{i}$. Let $\left\{q^{k}\right\}_{k \in \mathcal{K}}$ denote a solution to (1) then $v_{i}(\mathbf{x})$ is non-decreasing in $x_{i}$ for all $i \in \mathcal{I}, \mathbf{x} \in X$.

The proof can be found in the Appendix. The idea is to consider a partition of $[0,1]^{K|X|}$ and define a discrete approximation of the Bayesian mechanism $\left\{\tilde{q}^{k}\right\}_{k \in \mathcal{K}}$ by replacing the $\tilde{q}^{k}$ with their averages in each element of the partition. Lemma 1 ensures there exists an equivalent DIC mechanism $\left\{q^{k}\right\}_{k \in \mathcal{K}}$ for this discrete approximation. The $q^{k}$ can be extended to piecewise constant functions over $[0,1]^{K|X|}$. The result follows by considering increasingly finer partitions of $[0,1]$.

Lemma 3. Suppose, for all $i \in \mathcal{I}, X_{i} \subseteq \mathbb{R}$ and $\lambda_{i}$ is some distribution on $X_{i}$. Let $\left\{q^{k}\right\}_{k \in \mathcal{K}}$ denote a solution to (1) then $v_{i}(\mathbf{x})$ is non-decreasing in $x_{i}$ for all $i \in \mathcal{I}, \mathbf{x} \in X$. 
The proof can be found in the Appendix. The intuition is to consider a transformation of variables and relate the uniform distribution covered by Lemma 2 to the case of a general distribution. In particular, if the random variable $Z_{i}$ is uniformly distributed then $\lambda_{i}^{(-1)}\left(Z_{i}\right)$, with $\lambda_{i}^{(-1)}\left(z_{i}\right)=\inf \left\{x_{i} \in X_{i} \mid \lambda_{i}\left(x_{i}\right) \geq z_{i}\right\}$, is distributed according to $\lambda_{i}$.

We next establish that the interim expected utilities $u_{i}\left(x_{i}\right)$ in the DIC mechanism $(q, t)$ are the same as the interim expected utilities $\tilde{u}_{i}\left(x_{i}\right)$ in the BIC mechanism $(\tilde{q}, \tilde{t})$. Taking expectations over $\mathbf{x}_{-i}$ in 2 yields

$$
\begin{aligned}
\mathcal{T}_{i}\left(x_{i}\right) & =\tilde{\mathcal{T}}_{i}\left(\underline{x}_{i}\right)+V_{i}\left(\underline{x}_{i}\right) \underline{x}_{i}-V_{i}\left(x_{i}\right) x_{i}+\int_{\underline{x}_{i}}^{x_{i}} V_{i}(t) d t \\
& =\tilde{\mathcal{T}}_{i}\left(\underline{x}_{i}\right)+\tilde{V}_{i}\left(\underline{x}_{i}\right) \underline{x}_{i}-\tilde{V}_{i}\left(x_{i}\right) x_{i}+\int_{\underline{x}_{i}}^{x_{i}} \tilde{V}_{i}(t) d t \\
& =\tilde{u}_{i}\left(x_{i}\right)-\tilde{V}_{i}\left(x_{i}\right) x_{i}=\tilde{\mathcal{T}}_{i}\left(x_{i}\right)
\end{aligned}
$$

and, hence, $u_{i}\left(x_{i}\right)=V_{i}\left(x_{i}\right) x_{i}+\mathcal{T}_{i}\left(x_{i}\right)=\tilde{V}_{i}\left(x_{i}\right) x_{i}+\tilde{\mathcal{T}}_{i}\left(x_{i}\right)=\tilde{u}_{i}\left(x_{i}\right)$. Furthermore, expected transfers are the same under the BIC and DIC mechanisms since

$$
E_{\mathbf{x}}\left(t_{i}(\mathbf{x})\right)=E_{\mathbf{x}}\left(\tilde{t}_{i}(\mathbf{x})\right)+\sum_{k \in \mathcal{K}} c_{i}^{k}\left(E_{\mathbf{x}}\left(\tilde{q}^{k}(\mathbf{x})\right)-E_{\mathbf{x}}\left(q^{k}(\mathbf{x})\right)\right)=E_{\mathbf{x}}\left(\tilde{t}_{i}(\mathbf{x})\right)
$$

where we used that the ex ante expected probability with which each alternative occurs is the same under the BIC and DIC mechanisms, i.e. $E_{\mathbf{x}}\left(q^{k}(\mathbf{x})\right)=E_{\mathbf{x}}\left(\tilde{q}^{k}(\mathbf{x})\right)$ for all $k \in \mathcal{K}$, which is imposed as a constraint in the minimization problem (1). Expected social surplus is simply the sum of expected transfers plus the sum of the (ex ante) expected utilities of the agents, which is the same for the BIC and DIC mechanisms.

Q.E.D.

Note that Lemma 3 above applies to any distribution, not just continuous ones. We used the assumption of continuous type spaces only to invoke payoff equivalence, which allowed us to define the DIC transfers as in (2). To prove BIC-DIC equivalence more generally, we next provide an explicit construction of the DIC transfers when type spaces are discrete.

For each $i \in \mathcal{I}$ let $X_{i}=\left\{l_{i}^{1}, \ldots, l_{i}^{N_{i}}\right\}$. A mechanism $(\tilde{q}, \tilde{t})$ is BIC if and only if (i) for all $i \in \mathcal{I}$ and $l_{i} \in X_{i}, \tilde{V}_{i}\left(l_{i}\right)$ is non-decreasing in $l_{i}$ and (ii) the transfers satisfy

$$
\left(\tilde{V}_{i}\left(l_{i}^{n}\right)-\tilde{V}_{i}\left(l_{i}^{n-1}\right)\right) l_{i}^{n-1} \leq \tilde{\mathcal{T}}_{i}\left(l_{i}^{n-1}\right)-\tilde{\mathcal{T}}_{i}\left(l_{i}^{n}\right) \leq\left(\tilde{V}_{i}\left(l_{i}^{n}\right)-\tilde{V}_{i}\left(l_{i}^{n-1}\right)\right) l_{i}^{n}
$$

for $n=2, \ldots, N_{i}$. Similarly, a mechanism $(q, t)$ is DIC if and only if (i) for all $i \in \mathcal{I}$ and $\mathbf{l} \in X$, $\tilde{v}_{i}\left(l_{i}, \mathbf{l}_{-i}\right)$ is non-decreasing in $l_{i}$ and (ii) the transfers satisfy

$$
\left(v_{i}\left(l_{i}^{n}, \mathbf{l}_{-i}\right)-v_{i}\left(l_{i}^{n-1}, \mathbf{l}_{-i}\right)\right) l_{i}^{n-1} \leq \tau_{i}\left(l_{i}^{n-1}, \mathbf{l}_{-i}\right)-\tau_{i}\left(l_{i}^{n}, \mathbf{l}_{-i}\right) \leq\left(v_{i}\left(l_{i}^{n}, \mathbf{l}_{-i}\right)-v_{i}\left(l_{i}^{n-1}, \mathbf{l}_{-i}\right)\right) l_{i}^{n}
$$


for $n=2, \ldots, N_{i}$. Note that both the BIC and DIC incentive constraints are based on utility comparisons for adjacent types only, i.e. $l_{i}^{n-1}$ and $l_{i}^{n}$. In the Appendix we show that, with one-dimensional types, these necessary conditions are also sufficient (e.g. Vohra, 2011). This is not generally true with multi-dimensional types as we demonstrate in Section 5 .

Define the modified DIC transfers

$$
\tau_{i}\left(l_{i}^{n}, \mathbf{l}_{-i}\right)=\tilde{\mathcal{T}}_{i}\left(l_{i}^{1}\right)-\sum_{m=2}^{n}\left(v_{i}\left(l_{i}^{m}, \mathbf{l}_{-i}\right)-v_{i}\left(l_{i}^{m-1}, \mathbf{l}_{-i}\right)\right) \alpha_{i}^{m}
$$

for $n=1, \ldots, N_{i}{ }^{2} i \in \mathcal{I}$, and $\mathbf{l}_{-i} \in X_{-i}$, where

$$
\alpha_{i}^{n} \equiv \frac{\tilde{\mathcal{T}}_{i}\left(l_{i}^{n-1}\right)-\tilde{\mathcal{T}}_{i}\left(l_{i}^{n}\right)}{\tilde{V}_{i}\left(l_{i}^{n}\right)-\tilde{V}_{i}\left(l_{i}^{n-1}\right)}
$$

if $\tilde{V}_{i}\left(l_{i}^{n}\right) \neq \tilde{V}_{i}\left(l_{i}^{n-1}\right)$ and $\alpha_{i}^{n}=l_{i}^{n}$ otherwise. Note that the BIC constraints (3) imply that $l_{i}^{n-1} \leq \alpha_{i}^{n} \leq l_{i}^{n}$, which, in turn, implies that the difference in DIC transfers

$$
\tau_{i}\left(l_{i}^{n-1}, \mathbf{l}_{-i}\right)-\tau_{i}\left(l_{i}^{n}, \mathbf{l}_{-i}\right)=\left(v_{i}\left(l_{i}^{n}, \mathbf{l}_{-i}\right)-v_{i}\left(l_{i}^{n-1}, \mathbf{l}_{-i}\right)\right) \alpha_{i}^{n}
$$

satisfies the bounds in (4).

Now let $\left\{q^{k}\right\}_{k \in \mathcal{K}}$ denote a solution to minimization problem in (1). Lemma 1 ensures that the associated $v_{i}(\mathbf{l})$ is non-decreasing in $l_{i}$ for all $i \in \mathcal{I}, \mathbf{l} \in X$, and by construction $V_{i}\left(l_{i}\right)=E_{l_{-i}}\left(v_{i}\left(l_{i}, \mathbf{l}_{-i}\right)\right)=\tilde{V}_{i}\left(l_{i}\right)$. Taking expectations over $\mathbf{l}_{-i}$ in (5) yields

$$
\begin{aligned}
\mathcal{T}_{i}\left(l_{i}^{n}\right) & =\tilde{\mathcal{T}}_{i}\left(l_{1}^{n}\right)-\sum_{m=2}^{n}\left(V_{i}\left(l_{i}^{m}\right)-V_{i}\left(l_{i}^{m-1}\right)\right) \alpha_{i}^{m} \\
& =\tilde{\mathcal{T}}_{i}\left(l_{1}^{n}\right)+\sum_{m=2}^{n}\left(\tilde{\mathcal{T}}_{i}\left(l_{i}^{m}\right)-\tilde{\mathcal{T}}_{i}\left(l_{i}^{m-1}\right)\right)=\tilde{\mathcal{T}}_{i}\left(l_{i}^{n}\right)
\end{aligned}
$$

for $n=1, \ldots, N_{i}$. Hence, $u_{i}\left(l_{i}\right)=V_{i}\left(l_{i}\right) l_{i}+\mathcal{T}_{i}\left(l_{i}\right)=\tilde{V}_{i}\left(l_{i}\right) l_{i}+\tilde{\mathcal{T}}_{i}\left(l_{i}\right)=\tilde{u}_{i}\left(l_{i}\right)$, i.e. the DIC mechanism $(q, t)$ yields the same interim expected utilities as $(\tilde{q}, \tilde{t})$.

Theorem 2. Let $X_{i}$ be discrete for all $i \in \mathcal{I}$ and let $(\tilde{q}, \tilde{t})$ denote a BIC mechanism. An equivalent DIC mechanism is given by $(q, t)$, where the allocation rule $q$ solves (1) and the transfers follow from (5). Expected transfers and expected social surplus are the same under the BIC and DIC mechanisms.

\footnotetext{
${ }^{2}$ Where we use the convention that $\sum_{m=2}^{1} 1=0$.
} 
The fact that expected transfers and expected social surplus are the same follows from $\mathcal{T}_{i}\left(l_{i}\right)=$ $\tilde{\mathcal{T}}_{i}\left(l_{i}\right)$ for all $l_{i} \in X_{i}$ and the constraint, imposed in (1), that the ex ante expected probability with which each alternative occurs is the same under the BIC and DIC mechanisms.

\section{Relation to Other Papers}

In this section we illustrate our approach with some examples and relate our results to those of Manelli and Vincent (2010) for single-unit, private value auctions. First, we contrast our findings with those of Gershkov, Moldovanu, and Shi (2011) who present a counter example to DIC-BIC equivalence when there are three social alternatives.

\subsection{GMS Counter Example}

Following Gershkov, Moldovanu, and Shi (2011) consider the following example with $I=2$ agents and $K=3$ alternatives. Agent 1 has types $x_{1}<x_{2}$ and agent 2 has types $y_{1}<y_{2}$. For both agents, types are independent and equally likely. The utility (net of any transfers) of agent 1 with type $x_{i}$ is $a x_{i}+c$ in alternative $1, x_{i}+d$ in alternative 2 , and $v$ (independent of agent 1's type) in alternative 3. Agent 2's utilities simply follow by replacing the $x_{i}$ with $y_{i}$. It is assumed that $0<a<1$ with no restrictions on the constants $c, d$, and $v$. The utility parameters are summarized by the matrices

$$
A=\left(\begin{array}{lll}
a & 1 & 0 \\
a & 1 & 0
\end{array}\right), \quad C=\left(\begin{array}{lll}
c & d & v \\
c & d & v
\end{array}\right)
$$

Gershkov, Moldovanu, and Shi (2011) start with the following allocation rule

$$
\tilde{q}^{1}=s\left(\begin{array}{cc}
1 & 1 \\
1 & 13
\end{array}\right), \quad \tilde{q}^{2}=\text { as }\left(\begin{array}{ll}
9 & 1 \\
1 & 1
\end{array}\right)
$$

and $\tilde{q}_{i j}^{3}=1-\tilde{q}_{i j}^{1}-\tilde{q}_{i j}^{2}$ with $s=1 / 15$ and $\tilde{q}_{i j}^{k} \equiv \tilde{q}^{k}\left(x_{i}, y_{j}\right)$. It is easy to verify that the $\left\{\tilde{q}^{k}\right\}_{k \in \mathcal{K}}$ are Bayesian implementable since

$$
A \cdot \tilde{\mathbf{q}}=\text { as }\left(\begin{array}{cc}
10 & 2 \\
2 & 14
\end{array}\right)
$$

has non-decreasing marginals, i.e. $6 a s$ for the low type and 8 as for the high type. The $\left\{\tilde{q}^{k}\right\}_{k \in \mathcal{K}}$ are not dominant strategy implementable since $A \cdot \tilde{\mathbf{q}}$ is not everywhere non-decreasing. 
The question whether there is an equivalent DIC mechanism boils down to whether there exist $\mathbf{q}=\left(q^{1}, q^{2}, q^{3}\right)$ such that $A \cdot \mathbf{q}$ is everywhere non-decreasing and $A \cdot \mathbf{q}$ has the same marginals as $A \cdot \tilde{\mathbf{q}}$, i.e. $\sum_{k \in \mathcal{K}} a_{i}^{k} Q_{i}^{k}\left(x_{i}\right)=\sum_{k \in \mathcal{K}} a_{i}^{k} \tilde{Q}_{i}^{k}\left(x_{i}\right)$. Note that since $A$ does not have full rank, the equal-marginal condition does not require that $\mathbf{q}$ has the same marginals as $\tilde{\mathbf{q}}$. Indeed, Gershkov, Moldovanu, and Shi (2011) show that there exist no $\left\{q^{k}\right\}_{k \in \mathcal{K}}$ that satisfy $Q_{i}^{k}\left(x_{i}\right)=\tilde{Q}_{i}^{k}\left(x_{i}\right)$ and are such that $A \cdot \mathbf{q}$ is everywhere non-decreasing.

Their result seems to contradict Theorem 2. It does not, however, because there are other solutions to the condition that $A \cdot \mathbf{q}$ produces the same marginals as $A \cdot \tilde{\mathbf{q}}$ besides those considered by Gershkov, Moldovanu, and Shi (2011). Indeed, it is straightforward to solve the minimization problem in (1) to find $\left\{q^{k}\right\}_{k \in \mathcal{K}}$ that define an equivalent DIC mechanism:

$$
q^{1}=s\left(\begin{array}{ll}
4 & 6 \\
5 & 1
\end{array}\right), \quad q^{2}=a s\left(\begin{array}{ll}
1 & 1 \\
2 & 8
\end{array}\right)
$$

\subsection{Private-Value Auctions}

Manelli and Vincent (2010) recently proved equivalence of Bayesian and dominant strategy implementation for single-unit, private-value auctions. Their approach is similar to ours in some respects, e.g. their proof is constructive and covers the continuous case by considering a discrete approximation. There are, however, some differences. Most importantly, our analysis is not restricted to the single-unit auction case and includes multi-unit auctions for homogeneous and heterogeneous goods, combinatorial auctions, and the like. ${ }^{3}$ Indeed, applications of our BIC-DIC equivalence result extend beyond the auction context and include, for instance, public goods provision.

But also for single-unit auctions, our approach differs in several respects. First, Manelli and Vincent (2010) restrict attention to continuous distributions with connected supports. The discrete case covered by our Theorem 2 thus provides an important extension of their results. Moreover, Manelli and Vincent (2010) first prove BIC-DIC equivalence for the case with symmetric bidders (their Theorem 1), then introduce asymmetries between bidders (Theorem 2), and, finally, allow for the seller to have her own private value for the object (Theorem 3).

These different cases are all covered by the minimization approach in (1). To see this, consider a setup with $I+1$ agents ( $I$ bidders plus one seller) and $K=I+1$ alternatives. If the seller has no private value for the object we simply set $a_{i i}=1$ for $i=1, \ldots, I$ and $a_{i k}=0$ otherwise (and $\left.c_{i k}=0\right)$. By including the seller as the $(I+1)$-th agent, the possibility that the

\footnotetext{
${ }^{3}$ Assuming types are one-dimensional, independent, and private.
} 
object does not sell is included. In fact, the constraint $\sum_{k \in \mathcal{K}} q^{k}(\mathbf{x})=1$ in (1) becomes

$$
\sum_{k=1}^{I} q^{k}(\mathbf{x})=1-q^{I+1}(\mathbf{x})
$$

which combined with $E_{\mathbf{x}}\left(q^{k}(\mathbf{x})\right)=E_{\mathbf{x}}\left(\tilde{q}^{k}(\mathbf{x})\right)$ for all $k \in \mathcal{K}$ implies that if the seller does not sell with some probability in the original BIC mechanism then she does not sell with the same probability in the equivalent DIC mechanism. Furthermore, by including the seller as the $(I+1)$-th agent, the minimization approach in (1) implies that the constructed DIC mechanism generates the same expected revenue for the seller, since expected revenue is equal to the sum of bidders' expected payments. A fortiori, the DIC mechanism generates the same expected social surplus. To summarize, the constructed DIC mechanism is efficiency and revenue equivalent to the original BIC mechanism.

Moreover, if the original BIC mechanism is symmetric an equivalent symmetric DIC mechanism can be found by including symmetry as a constraint in (1) . Finally, the minimization approach in (1) also applies when the seller's private value is distributed over some range. In this case, we simply treat the seller like the bidders and set $a_{i i}=1$ for $i=1, \ldots, I+1$ and $a_{i k}=0$ otherwise.

To illustrate, consider a single-unit private value auction with $I=2$ bidders whose values, labeled $x_{1}$ and $x_{2}$, are independently and uniformly distributed on $[0,1]$. Suppose the seller does not allocate the object if the difference between bidders' values is too high, i.e. when $\left|x_{1}-x_{2}\right|>\alpha$ for some $\alpha \leq 1 / 25^{5}$ In all other cases, the seller allocates the object efficiently, see the left panel of Figure 1. Note that the allocation rule is not monotone and, hence, cannot be implemented in dominant strategies (Mookherjee and Reichelstein, 1992).

Denote the probability that bidder $k=1,2$ gets the object by $\tilde{q}^{k}$ and the probability that the seller keeps the object by $\tilde{q}^{3}$. So there are $K=3$ states, $a_{11}=a_{22}=1$ and $a_{i k}=0$ otherwise (and $c_{i k}=0$ ). For $i \neq j \in\{1,2\}$ the allocation rule can be stated as

$$
\tilde{q}^{i}(\mathbf{x})= \begin{cases}1 & \text { if } x_{j} \leq x_{i} \leq x_{j}+\alpha \\ 0 & \text { otherwise }\end{cases}
$$

\footnotetext{
${ }^{4}$ Note that the resulting constraint set is again non-empty and convex.

${ }^{5}$ For example, suppose the $x_{i}$ represent possible cost reductions from an innovation. Then a market regulator may prohibit the introduction of this innovation when the cost reductions are too asymmetric. In particular, if one firm's cost reduction is much larger than another's, the advantaged firm may be able to push the rival out of the market and gain monopoly power.
} 

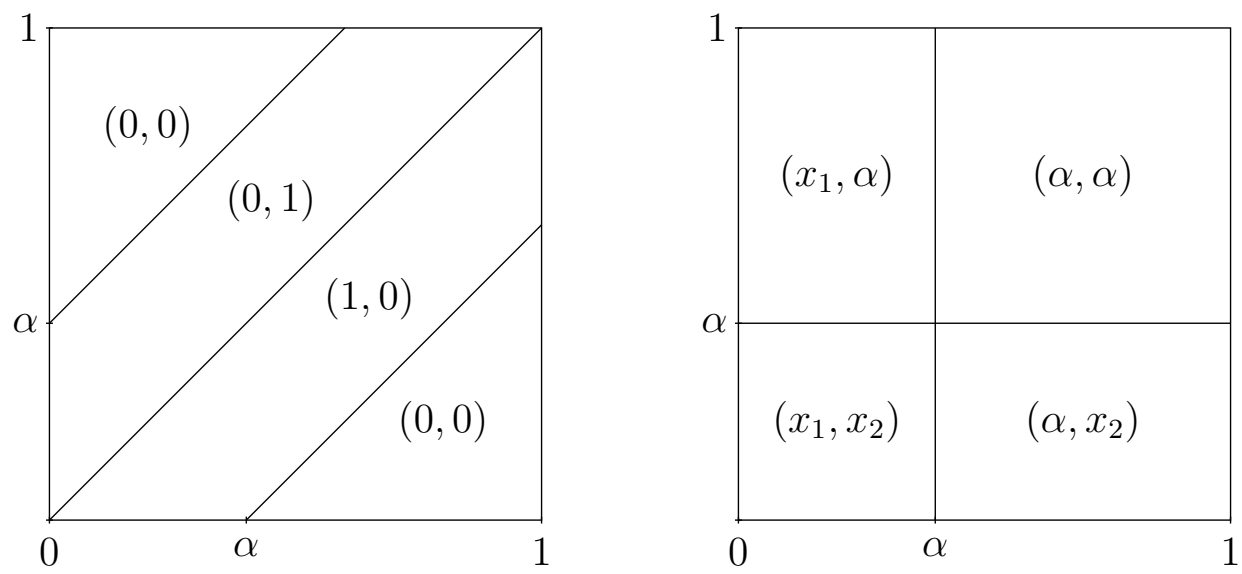

Figure 1: BIC allocation rule (left) and DIC allocation rule (right) for $\alpha \leq 1 / 2$. Here $\left(q_{1}, q_{2}\right)$ represent the probabilities that bidders $(1,2)$ win the object.

while $\tilde{q}^{3}(\mathbf{x})=1-\tilde{q}^{1}(\mathbf{x})-\tilde{q}^{2}(\mathbf{x})$. This allocation rule has non-decreasing marginals

$$
\int_{0}^{1} \tilde{q}^{i}(\mathbf{x}) d x_{j}=\min \left(x_{i}, \alpha\right)
$$

for $i \neq j \in\{1,2\}$, and is thus Bayesian implementable. For $\alpha \leq 1 / 2$ the allocation rule

$$
q^{i}(\mathbf{x})=\min \left(x_{i}, \alpha\right)
$$

for $i=1,2$ and $q^{3}(\mathbf{x})=1-\min \left(x_{1}, \alpha\right)-\min \left(x_{2}, \alpha\right)$ is a solution to minimization problem (1). This solution is shown in the right panel of Figure 1. Since the $\left\{q^{i}\right\}$ for $i=1,2$ are everywhere non-decreasing in $x_{i}$ they are dominant strategy implementable: supplemented with appropriate payments, they define an equivalent DIC mechanism.

\section{The Limits of BIC-DIC Equivalence}

In this section we present a series of simple examples, based on auction environments with two bidders and discrete types, which demonstrate that BIC-DIC equivalence generally does not hold when we relax the assumption of linear utilities or when types are not one-dimensional, private, and independent. Recall from the previous section that the constructed DIC mechanism is efficiency and revenue equivalent to the original BIC mechanism, which will prove useful in understanding the design of the counter-examples.

Denote the seller's expected revenue by $R$ and expected social surplus by $W$. Relaxing 
constraints in a revenue-maximization problem can only increase the achieved revenue level, so

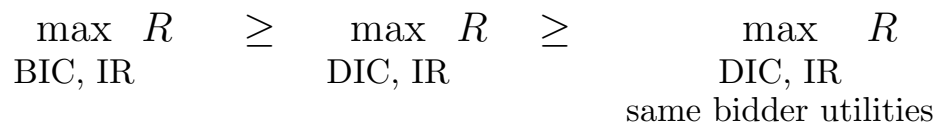

where IR, DIC, and BIC represent the individual rationality, dominant strategy incentive compatibility, and Bayesian incentive compatibility constraints respectively. For BIC-DIC equivalence to hold, these conditions have to be met with equality. $\left.\right|^{6}$ Conversely, if one of the conditions does not hold with equality, e.g. if the optimal DIC mechanism yields strictly less revenue than the optimal BIC mechanism, then BIC-DIC equivalence fails. A similar logic applies to social surplus, e.g. if the most efficient DIC mechanism yields strictly less welfare than the most efficient BIC mechanism then BIC-DIC equivalence fails.

\subsection{Interdependent Values}

Consider a discrete version of an example due to Maskin (1992). There are two bidders, labeled $i=1,2$, who compete for a single object. Bidder $i$ 's value for the object is $x_{i}+2 x_{j}$, where $i \neq j \in\{1,2\}$ and the signal $x_{i}$ is equally likely to be $x^{1}=1$ or $x^{2}=10$. Because of the higher weight on the other's signal, the first-best symmetric allocation rule is to assign the object to the lowest-signal bidder (with ties broken randomly)

$$
q^{1}=\left(\begin{array}{cc}
\frac{1}{2} & 1 \\
0 & \frac{1}{2}
\end{array}\right)
$$

and $q_{i j}^{2}=q_{j i}^{1}$ where we defined $q_{i j}^{k}=q^{k}\left(x_{1}^{i}, x_{2}^{j}\right)$ for $i, j, k=1,2$. The total surplus generated by the first-best allocation rule is $W=150 / 8$.

Maskin (1992) used a continuous version of this example to show that the first-best allocation rule is not Bayesian implementable. Here this follows simply because the marginals are decreasing in a bidder's signal. It is a simple linear programming problem to find the surplus-maximizing allocation rule that respects Bayesian incentive compatibility:

$$
q^{1}=\left(\begin{array}{cc}
0 & \frac{3}{4} \\
\frac{1}{4} & \frac{1}{2}
\end{array}\right)
$$

and $q_{i j}^{2}=q_{j i}^{1}$, yielding a total surplus of $W=135 / 8$. Note that this "second-best" allocation rule does not always assign the object and that the marginal probability of winning is constant.

\footnotetext{
${ }^{6} \mathrm{It}$ is important to point out that our BIC-DIC equivalence result in Section 3 is not constrained to revenuemaximizing BIC mechanisms. Here we limit attention to surplus-maximizing and revenue-maximizing BIC mechanisms only to derive conditions under which BIC-DIC equivalence fails.
} 
Importantly, the allocation rule is not monotone, so the second-best solution is not dominant strategy incentive compatible.7

For this example, the DIC mechanism that maximizes surplus is given by

$$
q^{1}=\left(\begin{array}{cc}
\frac{1}{2} & \frac{1}{2} \\
\frac{1}{2} & \frac{1}{2}
\end{array}\right)
$$

and $q_{i j}^{2}=q_{j i}^{1}$, yielding a total surplus of $W=132 / 8$. In other words, there exists no DIC mechanism that generates the same total surplus as the second-best solution in (6).

More generally, BIC-DIC equivalence breaks down with interdependent values even when the interdependencies are small. Suppose, for instance, that bidder $i$ 's value is $x_{i}+\alpha x_{j}$ for $i \neq j \in\{1,2\}$ and $\alpha \geq 0$. Consider the BIC mechanism in (6), which generates surplus $\left.W=\frac{3}{8}(11+17 \alpha)\right]^{8}$ The DIC allocation rule that gives the bidders the same interim expected utilities and maximizes surplus is given by

$$
q^{1}=\left(\begin{array}{cc}
\frac{1}{4} & \frac{1}{2} \\
\frac{1}{4} & \frac{1}{2}
\end{array}\right)
$$

and $q_{i j}^{2}=q_{j i}^{1}$, yielding a surplus of $W=\frac{3}{8}(11+14 \alpha)$. To summarize, only with private values $(\alpha=0)$, is it possible to construct an equivalent DIC mechanism.

\subsection{Correlated Values}

Cremer and McLean (1988, Appendix A) construct an example with correlated values for which a BIC mechanism extracts all surplus from the buyers while full-surplus extraction is not possible with a DIC mechanism.

\subsection{Multi-Dimensional Signals}

Consider a two-unit auction with $I=2$ bidders whose types are equally likely to be $x^{1}=(1,1)$, $x^{2}=(2,0)$, or $x^{3}=(4,3)$, where the first (second) number represents the marginal value for the first (second) unit. Note that marginal values are non-increasing for all three types, i.e. goods are substitutes. There are $K=3$ possible alternatives: $(0,2)$ where both units go to

\footnotetext{
${ }^{7}$ Hernando-Veciana and Michelucci (2009) previously demonstrated these properties for a continuous version of Maskin's (1992) example where the signals $x_{i}$ are uniformly distributed on $[0,1]$. They also provide a general characterization of second-best efficient mechanisms and show that, with two bidders, the second-best solution can be implemented via an English auction (Hernando-Veciana and Michelucci, 2011).

${ }^{8}$ Note that $[6]$ is not necessarily second-best for all $\alpha \geq 0$.
} 
bidder 2, $(1,1)$ where each bidder gets a unit, and $(2,0)$ where both units go to bidder 1 . It is straightforward to characterize the efficient allocation rule

$$
q^{1}=\left(\begin{array}{lll}
0 & 0 & 1 \\
0 & 0 & 1 \\
0 & 0 & 0
\end{array}\right), \quad q^{2}=\left(\begin{array}{lll}
1 & 1 & 0 \\
1 & 1 & 0 \\
0 & 0 & 1
\end{array}\right), \quad q^{3}=\left(\begin{array}{lll}
0 & 0 & 0 \\
0 & 0 & 0 \\
1 & 1 & 0
\end{array}\right)
$$

There are many marginal payments that support the efficient allocation rule as part of a BIC mechanism, e.g. $\left(\tilde{T}^{1}, \tilde{T}^{2}, \tilde{T}^{3}\right)=(0,0,1)$, resulting in interim expected utilities of $u\left(x^{1}\right)=\frac{2}{3}$, $u\left(x^{2}\right)=\frac{4}{3}$, and $u\left(x^{3}\right)=5$ for both bidders $5^{9}$ There is, however, no efficient DIC mechanism that gives the same interim expected utilities to the bidders. To see this, note that requiring the same interim expected utilities when using the same efficient allocation rule implies that the marginal payments are the same: $\frac{1}{3} \sum_{j} t_{i}^{1 j}=0, \frac{1}{3} \sum_{j} t_{i}^{2 j}=0$, and $\frac{1}{3} \sum_{j} t_{i}^{3 j}=1$ for $i=1,2$. Some of the dominant strategy incentive constraints for bidder $i$ when her type is $x_{i}$ and she faces an opponent of type $x_{j}$, to not report $\hat{x}_{i}$ are

$$
\begin{aligned}
& \left(x_{i}=x^{1}, x_{j}=x^{2}, \hat{x}_{i}=x^{3}\right) \quad: \quad t_{i}^{32} \geq 1+t_{i}^{12} \\
& \left(x_{i}=x^{1}, x_{j}=x^{1}, \hat{x}_{i}=x^{3}\right) \quad: \quad t_{i}^{31} \geq 1+t_{i}^{11} \\
& \left(x_{i}=x^{1}, x_{j}=x^{3}, \hat{x}_{i}=x^{2}\right) \quad: \quad t_{i}^{23} \geq t_{i}^{13} \\
& \left(x_{i}=x^{2}, x_{j}=x^{3}, \hat{x}_{i}=x^{3}\right) \quad: \quad t_{i}^{33} \geq 2+t_{i}^{23}
\end{aligned}
$$

Combining these constraints with the marginal payment conditions yields a contradiction

$$
3=t_{i}^{31}+t_{i}^{32}+t_{i}^{33} \geq 4+t_{i}^{11}+t_{i}^{12}+t_{i}^{23} \geq 4+t_{i}^{11}+t_{i}^{12}+t_{i}^{13}=4
$$

The difference with the one-dimensional case, and the reason that Theorem 2 does not apply, is that incentive compatibility requires more than just pairwise comparisons. With multidimensional types it is possible that, say, $x^{1}$ wants to deviate to $x^{3}$ even though $x^{1}$ does not want to deviate to $x^{2}$ and $x^{2}$ does not want to deviate to $x^{3}$. In the multi-dimensional type setting, Bayesian implementation imposes weaker conditions since some of the constraints that are violated under dominant strategy implementation may not be violated in expectation, i.e. when averaged over several types. Indeed, this is the case in the example presented here.

\footnotetext{
${ }^{9}$ To verify Bayesian incentive compatibility, note that when a bidder of type $x^{1}$ reports being of type $\hat{x}$ her interim expected utility is $\left(\frac{2}{3}, \frac{2}{3}, \frac{2}{3}\right)$ for $\hat{x}=\left(x^{1}, x^{2}, x^{3}\right)$. Likewise, for a bidder of type $x^{2}$ the interim expected utilities $\left(\frac{4}{3}, \frac{4}{3}, 1\right)$ for $\hat{x}=\left(x^{1}, x^{2}, x^{3}\right)$. Finally, for a bidder of type $x^{3}$ the interim expected utilities $\left(\frac{8}{3}, \frac{8}{3}, 5\right)$ for $\hat{x}=\left(x^{1}, x^{2}, x^{3}\right)$. Hence, truth telling $\hat{x}=x$ is an equilibrium.
} 
An interesting consequence is that with multi-dimensional types, BIC mechanisms may result in higher seller revenue than is possible under dominant strategy implementation. For example, consider a variant of the above setup with $x^{1}=(1,1), x^{2}=(2,0)$, and $x^{3}=(3,0)$. It is a standard linear-programming exercise to find the BIC mechanism that maximizes seller revenue:

$$
\tilde{q}^{1}=\left(\begin{array}{ccc}
\frac{1}{2} & 0 & 1 \\
1 & 0 & 0 \\
0 & 0 & 0
\end{array}\right), \quad \tilde{q}^{2}=\left(\begin{array}{ccc}
0 & 0 & 0 \\
0 & 1 & 1 \\
0 & 1 & 1
\end{array}\right), \quad \tilde{q}^{3}=\left(\begin{array}{ccc}
\frac{1}{2} & 1 & 0 \\
0 & 0 & 0 \\
1 & 0 & 0
\end{array}\right)
$$

supported by marginal payments $\left(\tilde{T}^{1}, \tilde{T}^{2}, \tilde{T}^{3}\right)=\left(1, \frac{4}{3}, \frac{7}{3}\right)$ and resulting in seller revenues of $R=28 / 9$. Now suppose we replace the BIC constraints with DIC constraints and we again maximize seller revenue without requiring that the DIC mechanism yields the same interim expected utilities for the bidders. In other words, we consider the optimal, i.e. revenuemaximizing, DIC mechanism. The solution for the allocation rule is the same as for the BIC case, i.e. $q^{k}=\tilde{q}^{k}$ for $k=1,2,3$, while the supporting payments are

$$
t_{1}=\left(\begin{array}{ccc}
1 & 0 & 0 \\
\frac{2}{3} & 0 & \frac{2}{3} \\
\frac{3}{2} & 0 & \frac{2}{3}
\end{array}\right), \quad t_{2}=\left(\begin{array}{ccc}
1 & \frac{2}{3} & \frac{3}{2} \\
0 & 0 & 0 \\
0 & \frac{2}{3} & \frac{2}{3}
\end{array}\right)
$$

resulting in seller revenues of $R=3$. In other words, the optimal DIC mechanism produces strictly less revenues than the optimal BIC mechanism, and BIC-DIC equivalence fails.

\subsection{Non-linear Utilities}

We can reinterpret the multi-dimensional type examples of the previous subsection in terms of non-linear utilities. Consider, for instance, the last example where a bidder's utility when her type is $x^{i}$ and the state is $k$, for $i, k=1,2,3$, are summarized by the matrix

$$
u_{k}^{i}=\left(\begin{array}{lll}
0 & 1 & 2 \\
0 & 2 & 2 \\
0 & 3 & 3
\end{array}\right)
$$

Let us introduce three one-dimensional types, $\theta^{1}=1, \theta^{2}=2$, and $\theta^{3}$, and, for both bidders, the non-linear utility functions $u^{k}$ for $k=1,2,3$, where $u^{1}(\theta)=0, u^{2}(\theta)=\theta$, and $u^{3}(\theta)=$ $\frac{1}{2} \theta^{2}-\frac{3}{2} \theta+3$. It is easy to verify that this non-linear model reproduces the utilities of the above matrix. Hence, bidders' interim expected utilities and their incentives to deviate are identical to those in the multi-dimensional example. A direct consequence is that again there is an optimal BIC mechanism that produces strictly higher revenues than is possible under DIC implementation. 


\section{Discussion}

This paper establishes a link between dominant strategy and Bayesian implementation for a general class of social choice problems. When utilities are linear and types are one-dimensional, independent, and private, we prove that for any social choice rule that is Bayesian implementable there exists a (possibly different) social choice rule that yields the same interim expected utilities for all agents and that is implementable in dominant strategies.

Dominant strategy implementation is more robust than Bayesian implementation since it does not rely on the assumptions of common prior beliefs and equilibrium play. In many settings, these assumptions are untenable. In contrast, dominant strategy implementation is robust to changes in agents' beliefs and allows agents to optimize without having to take into account others' behavior.

This paper also delineates the boundaries for BIC-DIC equivalence. When types are correlated, Cremer and McLean (1988) provide an example where a BIC mechanism yields strictly higher seller revenue than is attainable by any DIC mechanism. The example in Section 5.1 shows that with interdependent values, the expected social surplus generated by a BIC mechanism may be strictly higher than what can be generated by any DIC mechanism. When types are multi-dimensional or utilities are non-linear, BIC mechanisms may produce strictly higher seller revenue than is possible with DIC mechanisms, see the examples in Sections 5.3 and 5.4.

The equivalence of Bayesian and dominant strategy implementation thus requires linear utilities and one-dimensional, independent, and private values. When these conditions are met, Bayesian implementation provides no more flexibility than dominant strategy implementation in a general class of social choice problems. 


\section{A. Appendix}

Proof of Lemma 2. Let $C(\tilde{q})$ denote the collection of $\left\{q^{k}\right\}_{k \in \mathcal{K}}$ that satisfy the constraints in (1). Note that $C(\tilde{q})$ is non-empty since $\tilde{q} \in C(\tilde{q})$. Furthermore, $C(\tilde{q})$ is a non-empty, compact and convex subset of $\left\{f \in L^{\infty}\left([0,1]^{I}\right) \mid 0 \leq f \leq 1\right\}$ and $E_{\mathbf{l}}\left(\|\mathbf{v}(\mathbf{x})\|^{2}\right)$ defines a convex functional - again the solution to (1) exists 10 The intuition behind the proof is to relate this solution to that of Lemma 1 by taking a discrete approximation. For $i \in \mathcal{I}, n \geq 1, l_{i}=1, \ldots, 2^{n}$, define the sets $S_{i}\left(n, l_{i}\right)=\left[\left(l_{i}-1\right) 2^{-n}, l_{i} 2^{-n}\right)$, which yield a partition of $[0,1)$ into $2^{n}$ disjoint intervals of equal length. Let $\mathcal{F}_{i}^{n}$ denote the set consisting of all possible unions of the $S_{i}\left(n, l_{i}\right)$. Note that $\mathcal{F}_{i}^{n} \subset \mathcal{F}_{i}^{n+1}$. Also let $\mathbf{l}=\left(l_{1}, \ldots, l_{I}\right)$ and $S(n, \mathbf{l})=\prod_{i \in \mathcal{I}} S_{i}\left(n, l_{i}\right)$, which defines a partition of $[0,1)^{I}$ into disjoint half-open cubes of volume $2^{-n I}$. Let $\left\{\tilde{q}^{k}\right\}_{k \in \mathcal{K}}$ define a BIC mechanism and consider, for each $i \in \mathcal{I}$, the averages

$$
\begin{aligned}
\tilde{q}^{k}(n, \mathbf{l}) & =2^{n I} \int_{S(n, \mathbf{l})} \tilde{q}^{k}(\mathbf{x}) d \mathbf{x} \\
E_{\mathbf{l}_{-i}} \tilde{v}_{i}(n, \mathbf{l}) & =2^{n} \int_{S_{i}\left(n, l_{i}\right)} E_{\mathbf{x}_{-i}} \tilde{v}_{i}(\mathbf{x}) d x_{i}
\end{aligned}
$$

Since $\tilde{q}^{k}(\mathbf{x}) \geq 0$ and $\sum_{k} \tilde{q}^{k}(\mathbf{x})=1$ we have $\tilde{q}^{k}(n, \mathbf{l}) \geq 0$ and $\sum_{k} \tilde{q}^{k}(n, \mathbf{l})=1$. By construction $\sum_{\mathbf{l}_{-i}} \tilde{v}_{i}(n, \mathbf{l})=2^{n(I-1)} E_{\mathbf{l}_{-i}} \tilde{v}_{i}(n, \mathbf{l})$, which is non-decreasing in $l_{i}$ by A.2.

Lemma 1 applied to the case where, for each $i \in \mathcal{I}, X_{i}=\left\{1, \ldots, 2^{n}\right\}$ and $\lambda_{i}$ is the discrete uniform distribution on $X_{i}$, implies there exist $\left\{q^{k}(n, \mathbf{l})\right\}_{k \in \mathcal{K}}$ with $q^{k}(n, \mathbf{l}) \geq 0$ and $\sum_{k} q^{k}(n, \mathbf{l})=$ 1 such that $\sum_{\mathbf{l}_{-\mathbf{i}}} v_{i}(n, \mathbf{l})=\sum_{\mathbf{l}_{-\mathbf{i}}} \tilde{v}_{i}(n, \mathbf{l}), \sum_{\mathbf{l}} q^{k}(n, \mathbf{l})=\sum_{\mathbf{l}} \tilde{q}^{k}(n, \mathbf{l})$, and $v_{i}(n, \mathbf{l})$ is non-decreasing in $l_{i}$ for all $\mathbf{l}$.

For each $i \in \mathcal{I}, n \geq 1$ define $q^{k}(n, \mathbf{x})=q^{k}(n, \mathbf{l})$ for all $\mathbf{x} \in S(n, \mathbf{l})$. Then $q^{k}(n, \mathbf{x}) \geq 0$, $\sum_{k} q^{k}(n, \mathbf{x})=1$, and for each $i \in \mathcal{I}, v_{i}(n, \mathbf{x})$ is non-decreasing in $x_{i}$ for all $\mathbf{x}$. Furthermore

$$
\begin{gathered}
\int_{S_{i}\left(n, l_{i}\right)} E_{\mathbf{x}_{-i}} \tilde{v}_{i}(\mathbf{x}) d x_{i}=2^{-n} E_{\mathbf{l}_{-i}} \tilde{v}_{i}(n, \mathbf{l})=2^{-n I} \sum_{\mathbf{l}_{-i}} \tilde{v}_{i}(n, \mathbf{l})=2^{-n I} \sum_{\mathbf{l}_{-i}} v_{i}(n, \mathbf{l}) \\
=\sum_{l_{-i}} \int_{S(n, \mathbf{l})} v_{i}(n, \mathbf{x}) d \mathbf{x}=\int_{S_{i}\left(n, l_{i}\right) \times[0,1]^{I-1}} v_{i}(n, \mathbf{x}) d \mathbf{x}
\end{gathered}
$$

Thus $v_{i}(n, \mathbf{x})-E_{\mathbf{x}_{-\mathbf{i}}}\left(\tilde{v}_{i}(\mathbf{x})\right)$ integrates to 0 over every set $S_{i} \times[0,1]^{I-1}$ with $S_{i} \in \mathcal{F}_{i}^{n}$. Similarly $q^{k}(n, \mathbf{x})-\tilde{q}^{k}(\mathbf{x})$ integrates to 0 over every set $[0,1]^{I}$. Consider any (weak $\left.{ }^{*}\right)$ convergent subsequence from the sequence $\left\{q^{k}(n, \mathbf{x})\right\}_{k \in \mathcal{K}}$ for $n \geq 1$, with limit $\left\{q^{k}(\mathbf{x})\right\}_{k \in \mathcal{K}}$. Then $\left\{q^{k}(\mathbf{x})\right\}_{k \in \mathcal{K}}$ defines a DIC mechanism that is equivalent to $\left\{\tilde{q}^{k}(\mathbf{x})\right\}_{k \in \mathcal{K}}$.

Q.E.D.

Proof of Lemma 3. The intuition behind the proof is to relate the unique solution to (1) to that of the uniform case of Lemma 2. Recall that if the random variable $Z_{i}$ is uniformly

\footnotetext{
${ }^{10}$ See Gutmann et al. (1991, p. 1784). If the objective is a strictly convex functional the solution is unique.
} 
distributed then $\lambda_{i}^{-1}\left(Z_{i}\right)$ is distributed according to $\lambda_{i}{ }^{11}$ Hence, consider for all $i \in \mathcal{I}$ and $\mathbf{z} \in[0,1]^{I}$, the functions $\tilde{q}^{\prime k}(\mathbf{z})=\tilde{q}^{k}\left(\lambda_{1}^{-1}\left(z_{1}\right), \ldots, \lambda_{I}^{-1}\left(z_{I}\right)\right)$. Since

$$
E_{\mathbf{z}_{-i}}\left(\tilde{v}_{i}^{\prime}(\mathbf{z})\right)=E_{\mathbf{x}_{-i}}\left(\tilde{v}_{i}\left(\lambda_{i}^{-1}\left(z_{i}\right), \mathbf{x}_{-i}\right)\right)
$$

the mechanism defined by $\left\{\tilde{q}^{\prime k}\right\}_{k \in \mathcal{K}}$ is BIC and by Lemma 2 there exists an equivalent DIC mechanism $\left\{q^{\prime k}\right\}_{k \in \mathcal{K}}$ where $q^{\prime k}:[0,1]^{I} \rightarrow[0,1]$. In particular, $q^{\prime}$ minimizes $E_{\mathbf{z}}\left(\mathbf{v}(\mathbf{z})^{2}\right)$ and satisfies the constraints $q^{\prime k}(\mathbf{z}) \geq 0, \sum_{k} q^{\prime k}(\mathbf{z})=1$, and $E_{\mathbf{z}_{-i}}\left(v_{i}^{\prime}(\mathbf{z})\right)=E_{\mathbf{x}_{-i}}\left(\tilde{v}_{i}\left(\lambda_{i}^{-1}\left(z_{i}\right), \mathbf{x}_{-i}\right)\right)$ for all $i \in \mathcal{I}$. Now define $\left\{q^{k}\right\}_{k \in \mathcal{K}}$ with $q^{k}: X \rightarrow[0,1]$ where $q^{k}(\mathbf{x})=q^{\prime k}\left(\lambda_{1}\left(x_{1}\right), \ldots, \lambda_{I}\left(x_{I}\right)\right)$. Then $\left\{q^{k}\right\}_{k \in \mathcal{K}}$ solves (1) since $E_{\mathbf{x}}\left(\mathbf{v}(\mathbf{x})^{2}\right)=E_{\mathbf{z}}\left(\mathbf{v}^{\prime k}(\mathbf{z})^{2}\right)$ and $q^{k}(\mathbf{x}) \geq 0, \sum_{k} q^{k}(\mathbf{x})=1$, and $E_{\mathbf{x}_{-i}}\left(v_{i}(\mathbf{x})\right)=E_{\mathbf{z}_{-i}}\left(v_{i}^{\prime}\left(\lambda_{i}\left(x_{i}\right), \mathbf{z}_{-i}\right)\right)=E_{\mathbf{x}_{-i}}\left(\tilde{v}_{i}(\mathbf{x})\right)$ for all $i \in \mathcal{I}$ and $x_{i} \in X_{i}$. Furthermore, $v_{i}(\mathbf{x})=\sum_{k} a_{i k} q^{k}(\mathbf{x})=\sum_{k} a_{i k} q^{\prime k}\left(\lambda_{1}\left(x_{1}\right), \ldots, \lambda_{I}\left(x_{I}\right)\right)$ is non-decreasing in $x_{i}$ for all $k \in \mathcal{K}$, $x \in X$ since $\left\{q^{\prime}\right\}_{k \in \mathcal{K}}$ is a DIC mechanism, $\lambda$ is non-decreasing, and $a_{i k} \geq 0$.

Q.E.D.

Additional remarks to the proof of Theorem 2. For completeness, we show here that the necessary BIC (DIC) conditions (i) and (ii) are also sufficient. Consider (3) which ensures that deviating to an adjacent type, e.g. from $l_{i}^{n-1}$ to $l_{i}^{n}$, is not profitable. Now consider types $l_{i}^{p}<l_{i}^{q}<l_{i}^{r}$. We show that if it is not profitable for type $l_{i}^{p}$ to deviate to type $l_{i}^{q}$ and it is not profitable for type $l_{i}^{q}$ to deviate to type $l_{i}^{r}$ then it is not profitable for type $l_{i}^{p}$ to deviate to type $l_{i}^{r}$. The assumptions imply

$$
\tilde{V}_{i}\left(l_{i}^{p}\right) l_{i}^{p}+\tilde{\mathcal{T}}_{i}\left(l_{i}^{p}\right) \geq \tilde{V}_{i}\left(l_{i}^{q}\right) l_{i}^{p}+\tilde{\mathcal{T}}_{i}\left(l_{i}^{q}\right), \quad \tilde{V}_{i}\left(l_{i}^{q}\right) l_{i}^{q}+\tilde{\mathcal{T}}_{i}\left(l_{i}^{q}\right) \geq \tilde{V}_{i}\left(l_{i}^{r}\right) l_{i}^{q}+\tilde{\mathcal{T}}_{i}\left(l_{i}^{r}\right)
$$

and, hence,

$$
\tilde{V}_{i}\left(l_{i}^{p}\right) l_{i}^{p}+\tilde{\mathcal{T}}_{i}\left(l_{i}^{p}\right) \geq \tilde{V}_{i}\left(l_{i}^{r}\right) l_{i}^{p}+\tilde{\mathcal{T}}_{i}\left(l_{i}^{r}\right)+\left(\tilde{V}_{i}\left(l_{i}^{r}\right)-\tilde{V}_{i}\left(l_{i}^{q}\right)\right)\left(l_{i}^{q}-l_{i}^{p}\right) \geq \tilde{V}_{i}\left(l_{i}^{r}\right) l_{i}^{p}+\tilde{\mathcal{T}}_{i}\left(l_{i}^{r}\right)
$$

since $\tilde{V}_{i}\left(l_{i}\right)$ is non-decreasing and $l_{i}^{q}>l_{i}^{p}$. Similarly, we can show that if it is not profitable for type $l_{i}^{r}$ to deviate to type $l_{i}^{q}$ and it is not profitable for type $l_{i}^{q}$ to deviate to type $l_{i}^{p}$ then it is not profitable for type $l_{i}^{r}$ to deviate to type $l_{i}^{p}$. Finally, the same logic applies to the DIC constraints in (4). (Importantly, this derivation does not apply to multi-dimensional types as we show in Section 5.)

Q.E.D.

\footnotetext{
${ }^{11}$ Where $\lambda_{i}^{-1}\left(z_{i}\right)=\inf \left\{x_{i} \in X_{i} \mid \lambda_{i}\left(x_{i}\right) \geq z_{i}\right\}$.
} 


\section{References}

d'Aspremont, C. and L. A. Gérard-Varet (1979) "On Bayesian Incentive Compatible Mechanisms," in Aggregation and Revelation of Preferences, ed. by J.-J. Laffont, Amsterdam: North-Holland.

Crémer, J. and McLean. R. P. (1998) "Full Extraction of the Surplus in Bayesian and Dominant Strategy Auctions," Econometrica, 56(6), 1247-1258.

Fudenberg, D. and J. Tirole,(1991), Game Theory, MIT press.

Gershkov, A., B. Moldovanu, and X. Shi (2011) "Bayesian and Dominant Strategy Implementation Revisited," Working Paper, University of Bonn.

Gibbard, A. (1973) "Manipulation of Voting Schemes: a General Result," Econometrica, 41(4), 587-601.

Green, J. and J.-J. Laffont (1977) "Characterization of Satisfactory Mechanisms for the Revelation of Preferences for Public Goods," Econometrica, 45(2), 427-438.

Gutmann, S., J. H. B. Kemperman, J. A. Reeds, and L. A. Shepp (1991) "Existence of Probability Measures with Given Marginals," Annals of Probability, 19(4), 1781-1797.

Hernando-Veciana, A. and F. Michelucci (2009) "Second-Best Efficiency and the English Auction," Working paper, Universidad Carlos III de Madrid.

Hernando-Veciana, A. and F. Michelucci (2011) "Second-Best Efficiency and the English Auction," Games and Economic Behavior, forthcoming.

Heydenreich, B., R. Müller, M. Uetz, and R.V. Vohra (2009) "Characterization of Revenue Equivalence," Econometrica, 77(1), 307-316.

Jehiel, P., M. Meyer-ter-Vehn, and B. Moldovanu (2008) "Ex-post Implementation and Preference Aggregation via Potentials," Economic Theory, 37(3), 469-490.

Jehiel, P., M. Meyer-ter-Vehn, B. Moldovanu, and W.R. Zame (2006) "The Limits of Ex Post Implementation," Econometrica, 74(3), 585-610.

Jehiel, P. and B. Moldovanu (2001) "Efficient Design With Interdependent Valuations," Econometrica, 69(5), 1237-1259.

Jehiel, P., B. Moldovanu, and E., Stacchetti (1996) "How (Not) to Sell Nuclear Weapons," American Economic Review, 86(4), 814-829.

Jehiel, P., B. Moldovanu, and E., Stacchetti (1999) "Multidimensional Mechanism Design for Auctions With Externalities," Journal of Economic Theory, 85(2), 258-293.

Krishna, V. and E. Maenner (2001) "Convex Potentials With an Application to Mechanism Design," Econometrica, 69(4), 1113-1119. 
Laffont, J.-J. and E. Maskin (1979) "A Differential Approach to Expected Utility Maximizing Mechanisms," in Aggregation and Revelation of Preferences, ed. by J.-J. Laffont, Amsterdam: North-Holland.

Laffont, J.-J. and E. Maskin (1980) "A Differential Approach to Dominant Strategy Mechanisms," Econometrica, 48(6), 1507-1520.

Laffont, J.-J. and E. Maskin (1982) "The Theory of Incentives: an Overview," in Advances in Economic Theory, ed. by W. Hildenbrand, Cambridge University Press, 31-94.

Lavi, R., A. Mu'alem, and N. Nisan (2009) "Two simplified proofs for Roberts' theorem," Social Choice and Welfare, 32(3), 407-423.

Makowski, L. and C. Mezzetti (1994) "Bayesian and Weakly Robust First Best Mechanisms: Characterizations," Journal of Economic Theory, 64(2), 500-519.

Manelli, A. M. and D. R. Vincent (2010) "Bayesian and Dominant-Strategy Implementation in the Independent Private Values Model," Econometrica, 78(6), 1905-1938.

Mas-Colell, A., M. Whinston, and J. Green (1995), Microeconomic Theory, Oxford University Press.

Maskin, E. (1992) "Auctions and Privatization," in Privatization: Symposium in honour of Herbert Giersh, ed. by H. Siebert, Institute fur Weltwirtshaft an der Univarsitat Kiel.

Milgrom, P. and I. Segal (2002) "Envelope Theorems for Arbitrary Choice Sets," Econometrica, 70(2), 583-601.

Mookherjee, D. and S. Reichelstein (1992) "Dominant Strategy Implementation of Bayesian Incentive Compatible Allocation Rules," Journal of Economic Theory, 56(2), 378-399.

Myerson, R.B. (1981) "Optimal Auction Design," Mathematics of Operations Research, 6(1), $58-73$.

Myerson, R.B. and M.A. Satterthwaite (1983) "Efficient Mechanisms for Bilateral Trading," Journal of Economic Theory, 29(2), 265-281.

Roberts, K. (1979) "The Characterization of Implementable Choice Rules," in Aggregation and Revelation of Preferences, ed. by J.-J. Laffont, Amsterdam: North-Holland.

Satterthwaite, M.A. (1975) "Strategy-proofness and Arrow's Conditions: Existence and Correspondence Theorems for Voting Procedures and Social Welfare Functions," Journal of Economic Theory, 10(2), 187-217.

Vohra, R. V. (2011) Mechanism Design: A Linear Programming Approach, Cambridge University Press.

Williams, S. (1999) "A Characterization of Efficient, Bayesian Incentive Compatible Mechanisms," Economic Theory, 14(1), 155-180. 